\title{
\begin{tabular}{l|l} 
Mibraries & DSpace@MIT
\end{tabular}
}

\author{
MIT Open Access Articles
}

\section{A TRANSMISSION SPECTRUM OF TITAN'S NORTH POLAR ATMOSPHERE FROM A SPECULAR REFLECTION OF THE SUN}

The MIT Faculty has made this article openly available. Please share how this access benefits you. Your story matters.

Citation: Barnes, Jason W., Roger N. Clark, Christophe Sotin, Mate Adamkovics, Thomas Appere, Sebastien Rodriguez, Jason M. Soderblom, et al. "A TRANSMISSION SPECTRUM OF TITAN'S NORTH POLAR ATMOSPHERE FROM A SPECULAR REFLECTION OF THE SUN." The Astrophysical Journal 777, no. 2 (October 24, 2013): 161. (C) 2013 The American Astronomical Society

As Published: http://dx.doi.org/10.1088/0004-637x/777/2/161

Publisher: IOP Publishing

Persistent URL: http://hdl.handle.net/1721.1/94552

Version: Final published version: final published article, as it appeared in a journal, conference proceedings, or other formally published context

Terms of Use: Article is made available in accordance with the publisher's policy and may be subject to US copyright law. Please refer to the publisher's site for terms of use. 


\title{
A TRANSMISSION SPECTRUM OF TITAN'S NORTH POLAR ATMOSPHERE FROM A SPECULAR REFLECTION OF THE SUN
}

\author{
Jason W. Barnes ${ }^{1,11}$, Roger N. Clark ${ }^{2}$, Christophe Sotin $^{3}$, Máté Ádámkovics ${ }^{4}$, Thomas Appéré 5 , \\ Sebastien Rodriguez ${ }^{5}$, Jason M. SOderblom ${ }^{6}$, Robert H. Brown ${ }^{7}$, Bonnie J. Buratti ${ }^{3}$, \\ Kevin H. Baines ${ }^{8}$, Stéphane Le Mouélic ${ }^{9}$, And Philip D. Nicholson ${ }^{10}$ \\ ${ }^{1}$ Department of Physics, University of Idaho, Moscow, ID 83844-0903, USA; jwbarnes@uidaho. edu \\ 2 United States Geological Survey, Denver, CO 80225, USA

\begin{abstract}
Cassini/VIMS T85 observations of a solar specular reflection off of Kivu Lacus (87.4N 241.1E) provide an empirical transmission spectrum of Titan's atmosphere. Because this observation was acquired from short range $(33,000 \mathrm{~km})$, its intensity makes it visible within the $2.0,2.7$, and $2.8 \mu \mathrm{m}$ atmospheric windows in addition to the $5 \mu \mathrm{m}$ window where all previous specular reflections have been seen. The resulting measurement of the total one-way normal atmospheric optical depth (corresponding to haze scattering plus haze and gas absorption) provides strong empirical constraints on radiative transfer models. Using those models, we find that the total haze column abundance in our observation is $20 \%$ higher than the Huygens equatorial value. Ours is the first measurement in the $2-5 \mu \mathrm{m}$ wavelength range that probes all the way to the surface in Titan's arctic, where the vast majority of surface liquids are located. The specular technique complements other probes of atmospheric properties such as solar occultations and the direct measurements from Huygens. In breaking the degeneracy between surface and atmospheric absorptions, our measured optical depths will help to drive future calculations of deconvolved surface albedo spectra.
\end{abstract}

Key words: planets and satellites: individual (Titan) - radiative transfer - techniques: spectroscopic

Online-only material: color figures

\section{INTRODUCTION}

Difficulty in disambiguating surface and atmospheric contributions to infrared spectra impedes our understanding of the surface chemistry of Saturn's moon Titan. Cassini Visual and Infrared Mapping Spectrometer (VIMS; Brown et al. 2004) $0.35-5.12 \mu \mathrm{m}$ spectra of Titan show a combination of atmospheric and surface contributions within narrow spectral windows in the visible and near-visible (at 0.64, 0.68, 0.75, and $0.83 \mu \mathrm{m}$; Vixie et al. 2012) and near-infrared (at $0.93,1.08$, $1.28,1.58,2.0,2.7,2.8$, and $5.0 \mu \mathrm{m}$; e.g., Barnes et al. 2007). The resulting degeneracy hinders studies of both the surface and the atmosphere.

Several different approaches to resolving the degeneracy have been pursued previously. The Huygens probe deployed by Cassini actively measured solar spectra while descending through the atmosphere (Tomasko et al. 2005, 2008). Its data produced the most definitive measurements of Titan's atmospheric transmission and haze scattering properties. However, the Huygens profile was acquired at just one location and time, and its spectral range did not extend to wavelengths longer than $1.6 \mu \mathrm{m}$.

Comparison of observed spectra to forward radiative transfer modeling has also seen success. Using this technique, authors such as Rodriguez et al. (2006), Ádámkovics et al. (2009),

\footnotetext{
${ }^{11}$ Researcher ID: B-1284-2009
}

Bailey et al. (2011) Griffith et al. (2012), and Hirtzig et al. (2013) used increasingly sophisticated radiative transfer calculations to generate synthetic spectra that are then compared to either ground-based or spacecraft near-infrared spectra of Titan. This technique is potentially very powerful. However, it requires many inputs that are not always well known, including atmospheric absorption and haze scattering properties. When those values are not well known, residual surface/atmosphere degeneracies cannot be uniquely resolved.

Direct transmission spectra of Titan's atmosphere have been obtained by VIMS by watching the Sun disappear while Cassini flies behind Titan. This type of observation is called a solar occultation. Bellucci et al. (2009), Clark et al. (2010), Rannou et al. (2010), and C. Sotin et al. (2013b, in preparation) used VIMS solar occultations of the slant optical depth through a path parallel to a tangent to Titan's surface to infer the normal optical depth. This technique can be quite effective because Cassini has acquired eight solar occultations to date, at various times and latitudes. However, there have yet to be any near the north pole, and the slant optical path through Titan's atmosphere becomes high enough at shorter wavelengths so as to prevent probing of the lower atmosphere.

The Cassini Composite InfraRed Spectrometer has been used to probe Titan's arctic atmosphere at wavelengths longward of $5 \mu \mathrm{m}$. Vinatier et al. (2007) showed enhancement in the concentration of organic species at $80^{\circ} \mathrm{N}$ relative to $15^{\circ} \mathrm{S}$ from 


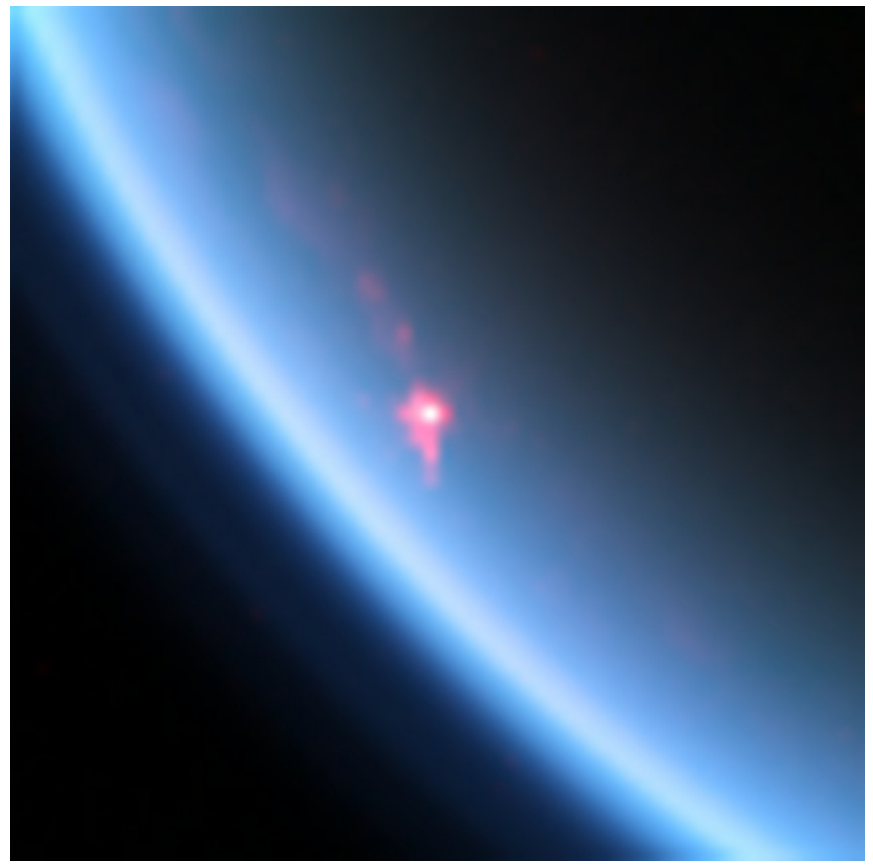

Figure 1. Specular reflection from T85, in cube CM_1721848119_1, in color with red mapped to $5 \mu \mathrm{m}$, green to $2.8 \mu \mathrm{m}$, and blue to $2.0 \mu \mathrm{m}$. The overall blue appearance of the image results from preferential scattering off of haze particles at shorter wavelengths at this high phase angle. This view shows some probable instrumental stray light in the $5 \mu \mathrm{m}$ channels (red) that makes the reflection look larger in red, as well as other semispecular reflections away from the specular point.

(A color version of this figure is available in the online journal.)

flybys in late 2004 and early 2005. And Teanby et al. (2010) showed increasing mixing ratios of trace gases at the north pole following spring equinox in 2009.

A perfectly diffuse reflector on the surface of Titan would make an excellent calibration target. Lacking such, the next best thing would be a perfectly specular reflector. Stephan et al. (2010) discovered specular reflections of the Sun off of Titan lakes ("sunglints") while Cassini looked back toward a crescent Titan at high phase angle. Barnes et al. (2011) used those data to constrain surface waves on one north polar lake (Jingpo Lacus), and Soderblom et al. (2012) described analytical and numerical models to calculate the expected intensities of specular reflections.

On T85, VIMS acquired its best and brightest specular reflection observation to date (Figure 1). In this paper, we use this specular reflection, visible at 5, 2.8, 2.7, and $2.0 \mu \mathrm{m}$, to empirically measure the transmissivity of Titan's atmosphere near the north pole. In Section 2 we describe the observation and its processing. We show the resulting transmission spectrum of Titan's atmosphere in Section 3. Finally, we discuss the implications of our work in Section 4, including comparison to both solar occultations and radiative transfer models, before concluding in Section 5.

\section{OBSERVATION}

The present work makes use of a single Cassini/VIMS spectral image cube: CM_1721848119_1. This cube was acquired on 2012 July 24 during the 85 th close Cassini flyby of Titan, T85 between 18:15:09 and 18:21:56 GMT. At that time, the spacecraft was $33,000 \mathrm{~km}$ above the surface of Titan. We used an exposure time of $80 \mathrm{~ms}$ per VIMS pixel. The cube has 64 pixels in both the $x$ (sample) and $y$ (line) directions-what we call a "full frame" cube. C. Sotin et al. (2013a, in preparation) describe how this particular T85 observation fits in with the rest of Cassini's Solstice Mission specular campaign.

We reduce the data to $I / F$ using the VIMS calibration pipeline, as described by Barnes et al. (2007), with one exception. Single, bright pixels across a few wavelengths get removed quite efficiently by an automated despiking algorithm that seeks to eliminate cosmic-ray hits. Therefore, automated despiking was turned off when processing this cube.

The pixel that shows the specular reflection is $(x=31$, $y=30$ ) using zero-offset array numbering (i.e., if the 64 pixels were numbered 0 through 63 ). The specular reflection is visible within the 2.0, 2.7, 2.8, and $5.0 \mu \mathrm{m}$ atmospheric spectral windows (Figure 2). It is not evident shortward of the $2.0 \mu \mathrm{m}$ window. Some specular counts appear shortward of the blue edge of the $5.0 \mu \mathrm{m}$ window (the window ends at $4.86 \mu \mathrm{m}$; Figure 2, lower-left). The unusual spatial structure of these pixels-bright in the three lines directly below the primary specular pixel-leads us to suspect that they may result from stray light within the instrument. Additionally, solar occultations show a strong $\mathrm{CO}$ absorption feature in this wavelength region (Lellouch et al. 2003; Baines et al. 2006; Clark et al. 2010) that does not appear in the VIMS T85 spectral data (we think because it is swamped out by stray light). Hence, we do not consider these channels when calculating our transmission spectrum.

We then isolate the purely spectral signal from that of surface reflection and forward-scattering off of haze particles. Of particular note are those pixels that show signal from specular skyglow-light specularly reflected off of the liquid surface in directions other than toward Cassini that gets forward-scattered by haze on the way out. We search the pixels in the cube for a suitable background measurement. For the primary specular pixel ( $x=31, y=30$; see Figure 3 ) we take the skyglow pixel to the left as the background $(x=30, y=30)$. When analyzing the skyglow pixel itself, we look for the pixel that is closest to the skyglow pixel in terms of incidence angle, emission angle, and phase angle. In this particular case, the reference background pixel for the skyglow pixel $(x=30, y=30)$ used to recreate the saturated wavelengths (see below) the reference background pixel is $(x=24, y=22)$. We then subtract the $I / F$ of this comparison signal from that of the specular pixel at each wavelength, resulting in a spectrum with purely specular contribution. The calibrated spectra for each of these three pixels are shown in Figure 4. The final spectrum thus gets generated from the calibrated VIMS data as

$$
\frac{I}{F}_{\text {specular }}=\frac{I}{F}_{(31,30)}-\frac{I}{F}_{(30,30)}
$$

for non-saturated data.

The specular pixel is saturated in two channels within the $2.8 \mu \mathrm{m}$ window and within all channels in the $5.0 \mu \mathrm{m}$ window. Therefore we attempt to recreate the saturated pixels by using the specular skyglow pixel $(x=30, y=30)$ one sample to the left of the specular pixel as a proxy. We use the backgroundsubtracted versions of both the specular pixel and the skyglow pixel $(x=30, y=30)$, and assume that the specular reflection value in the specular pixel ought to be that of the skyglow pixel times the specular/skyglow ratio in the nearest non-saturated channel. For saturated data points, we thus reconstruct the flux 


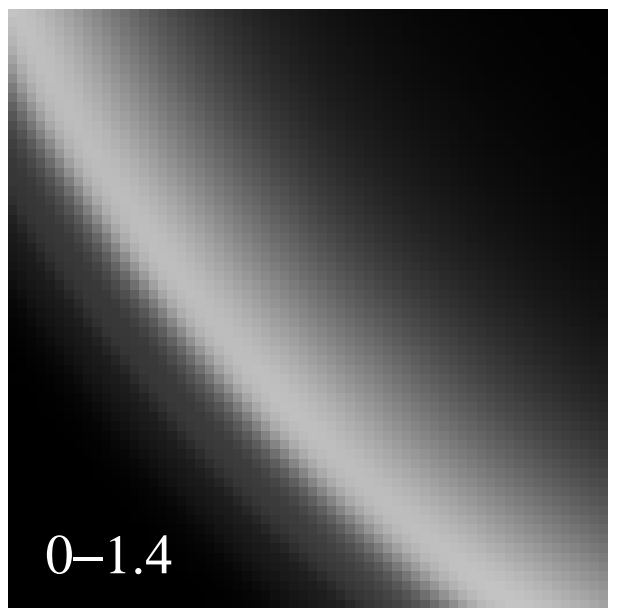

$1.59 \mu \mathrm{m}$

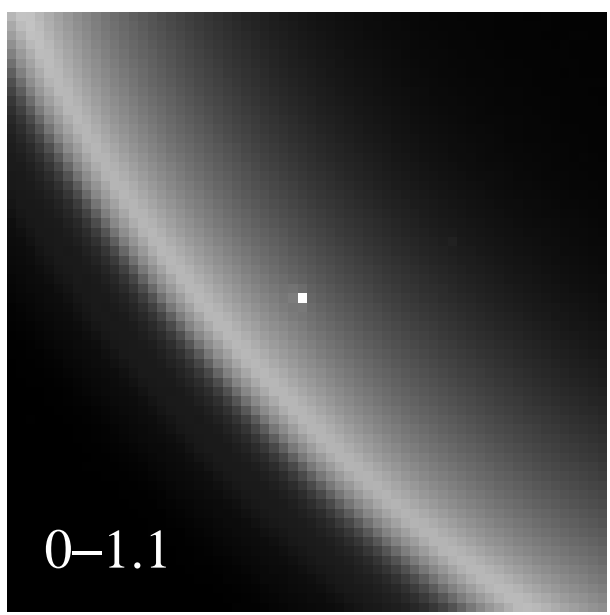

$2.70 \mu \mathrm{m}$

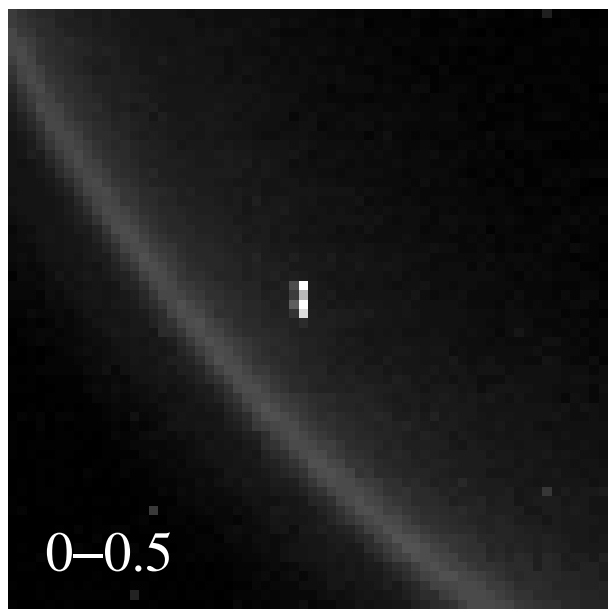

$4.74 \mu \mathrm{m}$

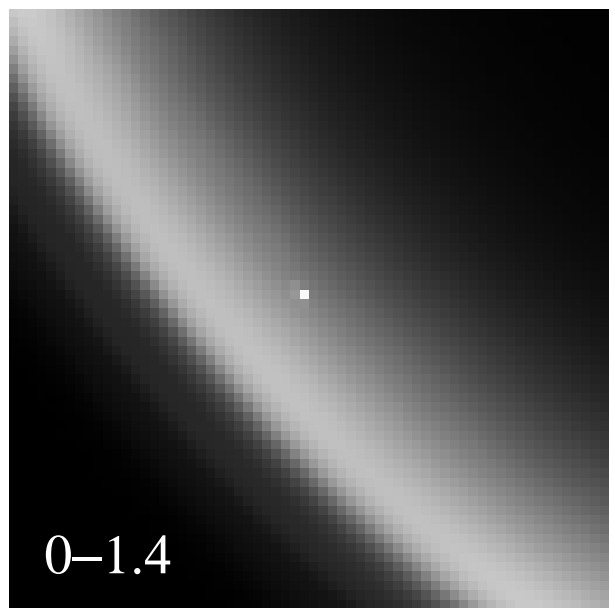

$2.00 \mu \mathrm{m}$

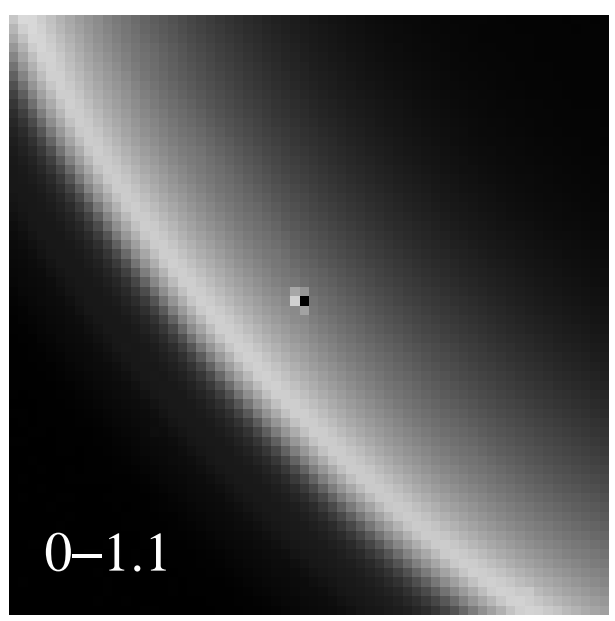

$2.78 \mu \mathrm{m}$

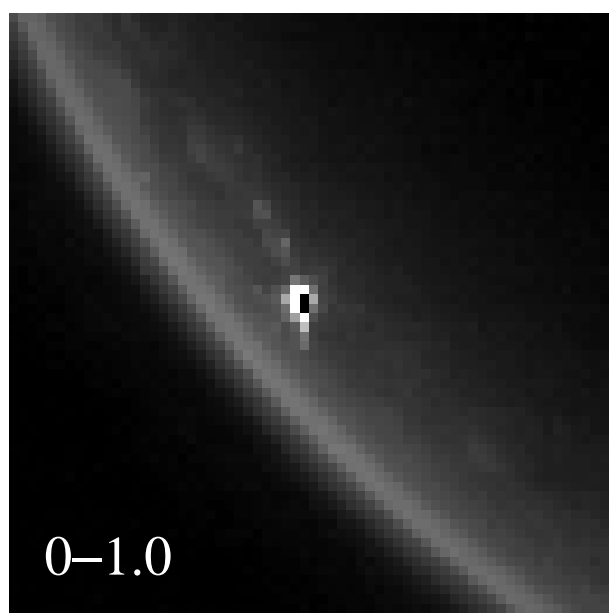

$4.94 \mu \mathrm{m}$

Figure 2. Spectral mapping images from VIMS cube CM_1721848119_1 at 1.6, 2.0, 2.70, 2.78, 4.74, and $4.94 \mu$ m wavelengths. The stretch used on each individual image is indicated in white text at the bottom left, to facilitate intercomparison. The black pixels at the core of the specular reflection at 2.78 and $4.94 \mu \mathrm{m}$ indicate saturation. The specular reflection is discernable at $2.00,2.70,2.78$, and $4.94 \mu \mathrm{m}$. It is not evident in the $1.59 \mu \mathrm{m}$ window. The brightness that surrounds the specular pixel at $5 \mu \mathrm{m}$ is specular skyglow: light specularly reflected off of Kivu Lacus in a direction other than toward Cassini that is later scattered toward Cassini off of haze. The apparent spatial distribution of the bright pixels below the specular point at $4.74 \mu \mathrm{m}$ is not consistent with any astrophysical effect. It is an instrumental artifact—possibly ghosting or scattered light within the instrument—and thus we consider as suspect those data shortward of the $4.8 \mu \mathrm{m}$ and longward of $4.0 \mu \mathrm{m}$ (see text and Figure 3). At $4.94 \mu \mathrm{m}$ the ghosting is still evident below the specular pixel; this is not a saturation effect. 


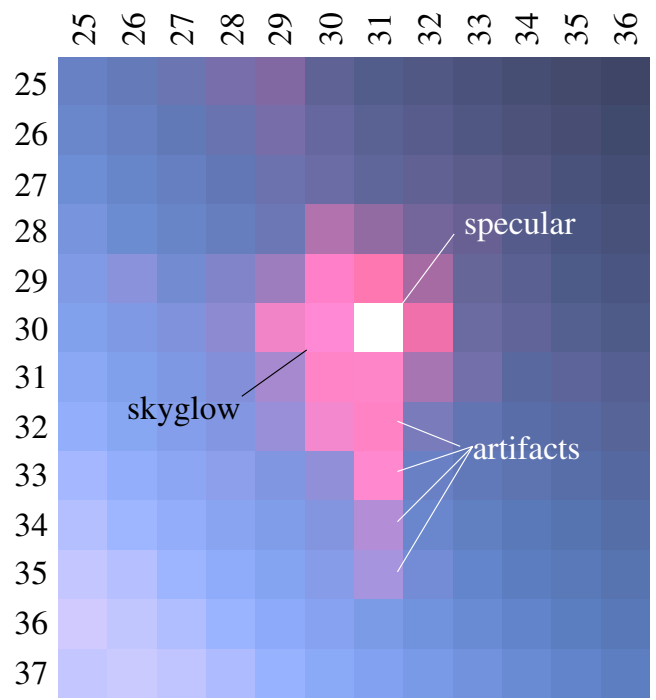

Figure 3. Annotated subwindow of VIMS cube CM_1721848119_1 showing the specular pixel and the areas bright at $5 \mu \mathrm{m}$ due to specular skyglow. The skyglow results from light specularly reflected off of the lake and then scattered toward Cassini by atmospheric haze.

(A color version of this figure is available in the online journal.)

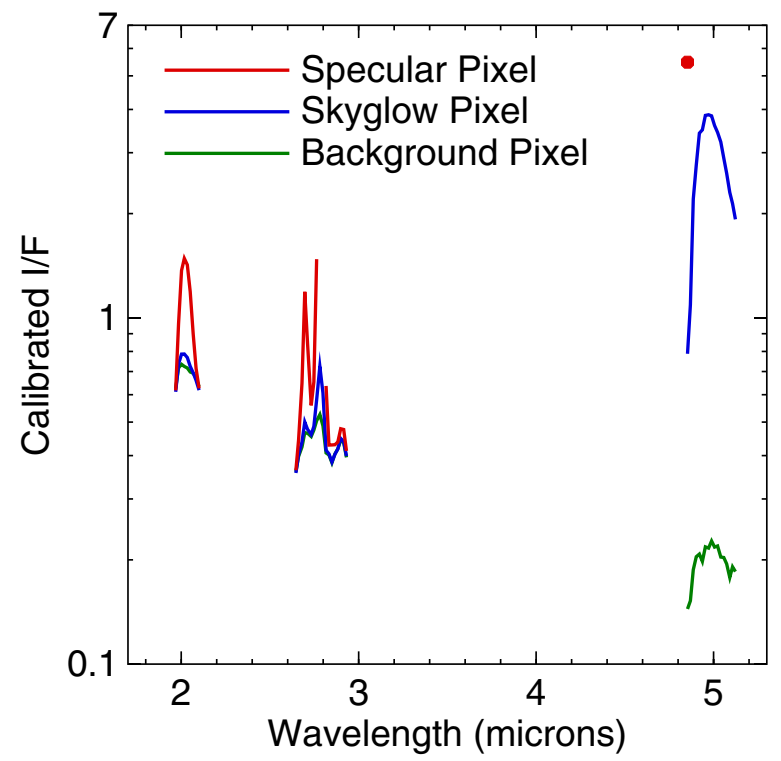

Figure 4. Calibrated $I / F$ spectra for the specular point, the specular skyglow pixel to the left of the specular point, and a background pixel. These are the raw inputs to Equations (1) and (2). The specular pixel (red) is saturated through most of the $5 \mu \mathrm{m}$ window and at the $2.8 \mu \mathrm{m}$ peak, so it has no values plotted there.

(A color version of this figure is available in the online journal.)

to be

$$
\begin{aligned}
\frac{I}{F}_{\text {specular }}= & \left(\frac{I}{F}_{(30,30)}-\frac{I}{F}_{(24,22)}\right) \\
& \left.\times \frac{\left(\frac{I}{F(31,30)}-\frac{I}{F}(30,30)\right)}{\left(\frac{I}{F}(30,30)\right.}-\frac{I}{F}(24,22)\right)_{\text {unsaturated }}
\end{aligned}
$$

in the saturated case.

The resulting shape for the $5.0 \mu \mathrm{m}$ window (see Section 3) matches well that from earlier flybys (e.g., T58; Soderblom et al. 2012). Thus we think that this saturation reconstruction is valid, though it likely introduces some small systematic errors. Acquiring specular reflection cubes using shorter integration times could solve the saturation problem for future observations.

A calculation of the precise specular location during T85 (following Soderblom et al. 2012) shows that the specular point was at $87.4 \mathrm{~N} 241.1 \mathrm{E}$. When the T85 VIMS observations are mapped and reprojected, the geographical location of the T85 specular reflection can be traced to a RADAR-dark lake called Kivu Lacus. In the left-hand portion of Figure 5, a north polar stereographic view of the Titan RADAR data shows that this area is generally somewhat RADAR-rough land surrounding $77.5 \mathrm{~km}$ diameter Kivu Lacus. The right-hand side of Figure 5 shows a reprojection of the $2 \mu \mathrm{m}$ VIMS view shown at the upper-right of Figure 2 for location comparison.

\section{TRANSMISSION}

The key insight of this paper is that the specular reflector on Titan's surface, in our case Kivu Lacus, acts as a mirror, reflecting the Sun's light according to Snell's Law. Snell's law depends on the index of refraction of both the air and the lake, which could theoretically vary between the atmospheric windows (Soderblom et al. 2012). However Titan's inferred composition of the lakes is liquid methane plus ethane (Brown et al. 2008), and we know that the 2, 2.7, 2.8, and $5 \mu \mathrm{m}$ windows do not include the fundamentals for these compounds. Therefore the real indices of refraction of liquid methane and other hydrocarbons show negligible wavelength dependence across the VIMS wavelength region, a result verified by laboratory measurements (Martonchik \& Orton 1994).

Thus, as sunlight passes through atmosphere on the way in, it is reflected off of the specular surface and traverses the atmosphere on the way out; the result is a transmission spectrum of Titan's atmosphere. On the way in, light that is absorbed by the haze or gases and light that is scattered by the haze gets removed from the beam and does not reflect off of the lake. Once light reaches the lake surface, a fraction of it reflects. The fraction that reflects is a function of the incidence angle and the index of refraction of the medium. The light beam is also altered by its reflection off of a convex surface-like your car mirror, objects seen in specular reflection off of a Titan lake are larger than they appear (Soderblom et al. 2012). Then light can be either absorbed or scattered again on the way out, which also removes it from the direct beam. Ultimately, then, the total amount of flux measured $I$ as divided by that that would be measured from a Lambertian surface at Titan's distance $F$ becomes

$$
\frac{I}{F}=\left(\frac{I}{F}\right)_{\text {specular }}(i, \eta, d) * e^{-2 \alpha(i)\left(\tau_{\mathrm{ga}}+\tau_{\mathrm{hs}}+\tau_{\mathrm{ha}}\right)}
$$

where $\tau_{\mathrm{ga}}, \tau_{\mathrm{hs}}$, and $\tau_{\mathrm{ha}}$ are the one-way normal optical depths of Titan's atmosphere for gaseous absorption, haze scattering, and haze absorption, respectively. We will refer to them later as $\tau=\tau_{\mathrm{ga}}+\tau_{\mathrm{hs}}+\tau_{\mathrm{ha}} .(I / F)_{\text {specular }}$ is the expected no-atmosphere intensity of the specular reflection, which is a function of the incidence angle $i$, the refractive index of the lake $\eta$, and the instantaneous distance between the spacecraft and the lake $d$, as described in Soderblom et al. (2012). Note that for specular reflections observed with low $d,(I / F)_{\text {specular }}$ can and does exceed 1.0 when normalized to $I / F$. The factor of two in the exponent arises from traversing the atmosphere in two directions, in and out.

The $\alpha$ in the exponent in Equation (3) corresponds to the equivalent number of normal atmospheres traversed by a photon 

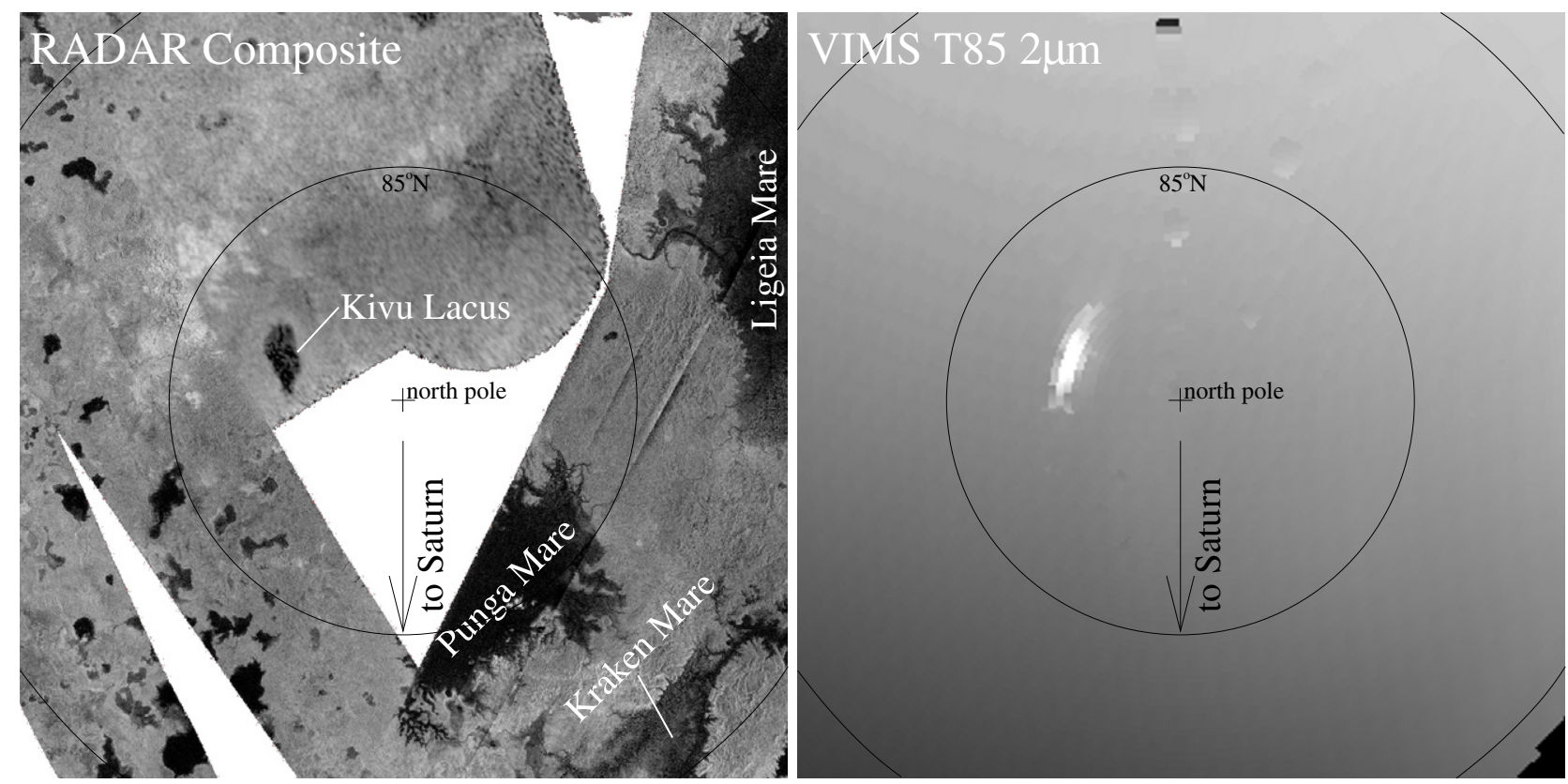

Figure 5. Two polar projection views of Titan's north pole. At left is the view from multiple Cassini RADAR passes, composited together in order to give context. The white areas are places with no RADAR coverage. At the right is a $2 \mu \mathrm{m}$ VIMS image view of the T85 cube that we analyze in this paper. The location of the bright specular reflection corresponds to the RADAR location of the lake named Kivu Lacus. The specular point on the T85 observation was at 87.4N 241.1E.

with incidence (or emission) angle $i$. Remember that in the specular case, the incidence angle is equal to the emission angle by definition. For a plane-parallel atmosphere, $\alpha(i)=$ $(1 / \cos i)$. However at high incidence and emission angles, Titan's real extended atmosphere differs significantly from the plane-parallel assumption. Hence we numerically calculate the number of atmospheres traversed as a function of emission angle, the airmass, using an explicit numerical integration of the straight path of a photon through Titan's spherical atmosphere.

Functionally we integrate over the height $h$ above Titan's surface, from the surface $(h=0)$ to the outer edge of the atmosphere $\left(h_{\max }\right)$. We then correct for the increased path length relative to zero incidence at each height by dividing by $\theta$, the angle between the photon's path and the surface beneath the point in question. The result is then normalized by the normal airmass:

$$
\alpha(i)=\frac{\int_{0}^{h \max } \frac{\rho(h)}{\cos (\theta(h, i))} d h}{\int_{0}^{h \max } \rho(h) d h}
$$

where

$$
\theta=\arcsin \left(\frac{r}{r+h} \sin (\pi-i)\right)
$$

from the Law of Sines (see Figure 6).

Figure 7 shows the numerical result for $\alpha(i)$ in a spherical atmosphere using two atmospheric structures: that of the Huygens HASI atmospheric density profile (Fulchignoni et al. 2005) and a hypothetical $150 \mathrm{~km}$ thick uniform atmosphere that we call an "orange rind." The incidence angle of the T85 Kivu specular reflection is $74^{\circ} .17$. Therefore the geometric factor $\alpha(74.17)$ for the T85 observation using the HASI profile is 2.517 , versus 3.666 for a plane-parallel atmosphere-a difference of $30 \%$.

Use of the HASI-measured density profile assumes that opacity is proportional to density, which is not precisely the case. In particular, haze structure can be complex and does not always mirror that of the gas, and methane (a prominent absorber) is not uniformly mixed throughout the atmosphere. A further complication is that refraction changes the inbound and outbound paths

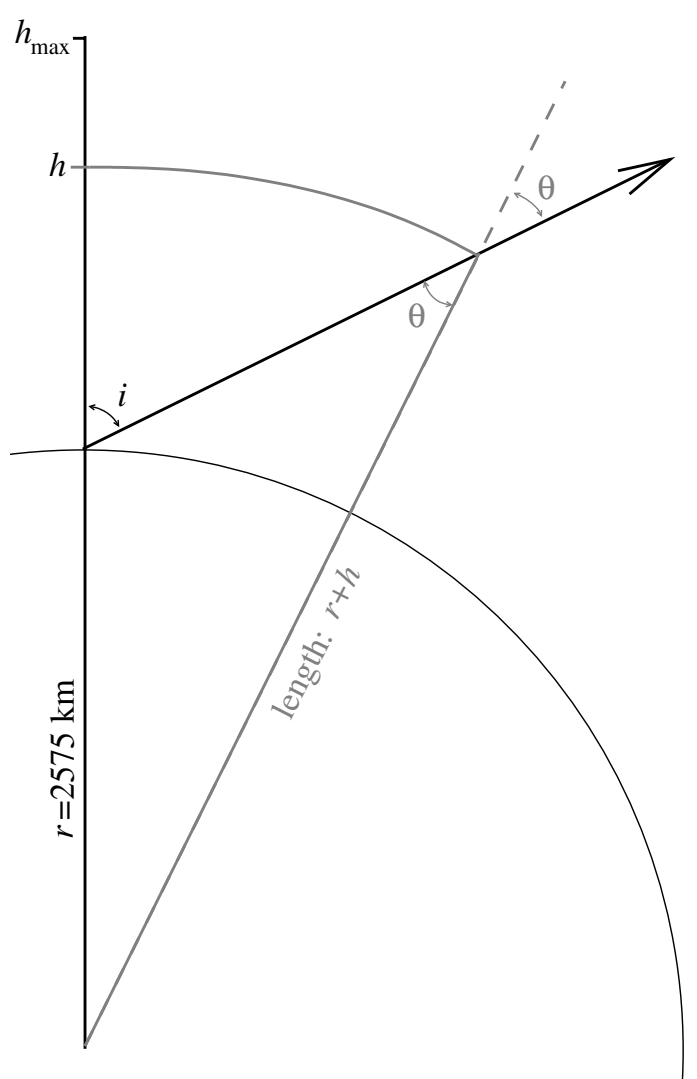

Figure 6. Geometry that we use for the numerical integral of opacity. Specifically, from the lower triangle we use the Law of Sines to derive Equation (5).

to not be straight lines through the atmosphere-a situation for which we do not account. However we do think that the spherical HASI correction ultimately provides a higher fidelity to the geometric correction than would a raw $\cos (i)$ plane-parallel 


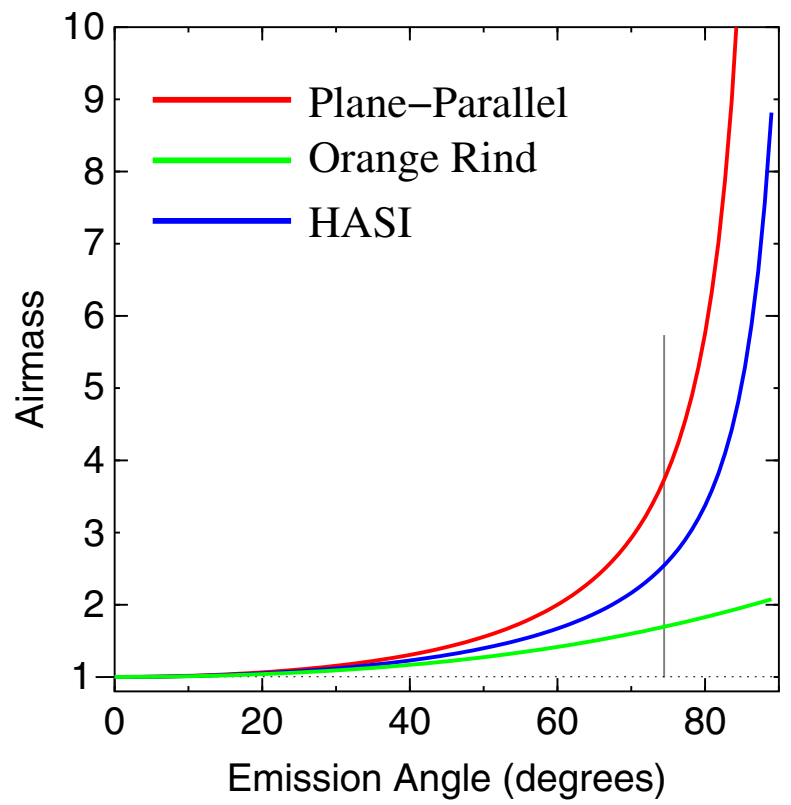

Figure 7. Path length for a photon encountering Titan's surface with a given emission (or incidence) angle, normalized to the normal atmospheric path with $e=0^{\circ}$. The red line indicates the number of traversed atmospheres under the plane-parallel assumption. Blue and green represent the number of traversed atmospheres as calculated using a numerical integration with Titan's parameters. The blue line derives from using the HASI atmospheric density profile (Fulchignoni et al. 2005) for the numerical integration, while the green line uses an "orange rind" atmosphere that has uniform density up to $150 \mathrm{~km}$. The vertical gray line indicates the emission angle of the T85 Kivu Lacus specular observation, 74.17. The orange rind formulation is included here solely for comparison purposes - all calculations in this paper use the HASI gas distribution, and assume that the haze distribution follows that of the gas.

(A color version of this figure is available in the online journal.)

correction. Future analyses may elect to use a more sophisticated approach.

This formulation also assumes that the total opacity is roughly constant within each VIMS spectel. Very sharp saturated absorption lines, for instance, would lead to a total measured absorption optical depth that was independent of emission angle. Under that scenario, our approach would underestimate the total optical depth once we have corrected for atmospheric path length. Future observations at multiple emission angles would allow us to measure this effect and compensate for it.

To infer Titan's transmission spectrum, then, we use the VIMS-measured background-subtracted and reconstructed $I / F$ of the specular pixel as the baseline (Figure 8, right-hand scale). We do this at every wavelength where the specular reflection is clearly evident in imaging and is not contaminated by stray light in the instrument. That measured intensity spectrum should be equal to the ideal specular intensity without atmosphere, minus the total light absorbed or scattered through the paths on the way in and out. Therefore

$$
\tau=\tau_{\mathrm{ga}}+\tau_{\mathrm{hs}}+\tau_{\mathrm{ha}}=-\frac{1}{2 \alpha(i)} \ln \left(\frac{I / F}{\left(\frac{I}{F}\right)_{\text {specular }}}\right)
$$

or alternatively

$$
\tau=-\frac{1}{2 \alpha(i)} \ln (I / F)+\frac{1}{2 \alpha(i)} \ln \left(\left(\frac{I}{F}\right)_{\text {specular }}\right)
$$

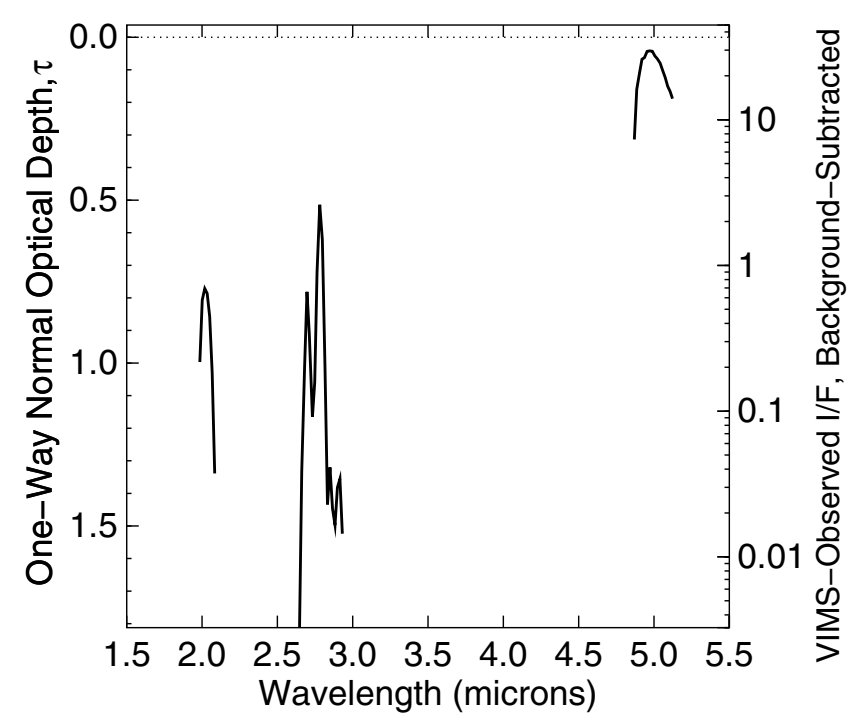

Figure 8. Inferred Titan transmission spectrum in two ways: using the scale at right, it is the VIMS-measured $I / F$ (background-corrected as described in Section 2), shown in a log scale; at left, it is the one-way normal optical depth for Titan's atmosphere as a function of wavelength, but note the inverted scale with $\tau=0.0$ at the top and $\tau=1.1$ at the bottom. We also show the raw optical depths used to generate this plot in Table 1.

or even

$$
\tau=-\frac{1}{2 \alpha(i)} \ln (I / F)+\tau_{\text {offset }}
$$

where $\tau_{\text {offset }}=(1 / 2 \alpha(i)) \ln \left((I / F)_{\text {specular }}\right)$ is a constant offset that slides the values in a $\tau$ versus $\lambda$ graph up or down. The $\tau_{\text {offset }}$ value is a function of $(I / F)_{\text {specular }}$ or the expected $I / F$ of the observed specular reflection without any atmospheric absorption.

The utility of the $\tau_{\text {offset }}$ formulation becomes clear as we consider potential sources of systematic error in our measurement. Small errors in $(I / F)_{\text {specular }}$, for instance, will cause vertical offsets in the measured optical depth spectrum by affecting $\tau_{\text {offset }}$. We know that some such error must exist based on our incomplete knowledge of the index of refraction $\eta$, which depends on the precise composition of Kivu Lacus. Though Ontario Lacus has been shown to contain ethane (Brown et al. 2008), the methane/ethane ratio, amount of dissolved nitrogen, and composition of dissolved organic solids, are all unknown and can potentially affect the overall value of $\eta$ by up to 0.1 , as $\eta_{\text {methane }}=$ 1.2872 while $\eta_{\text {ethane }}=1.3887$ (the resulting $\eta$ is still independent of wavelength; Badoz et al. 1992).

Note that the specular reflectivity of the surface is only a function of the real index of refraction-the absorption within the liquid quite specifically does not matter. We show a graphical representation of the index of refraction measurements for methane liquid from Martonchik \& Orton (1994) in Figure 9. The methane index of refraction is well-behaved over the wavelength range explored by VIMS, though measurements with tighter sampling and fewer gaps might be warranted in the future. The curve for ethane is expected to be similar. Although dissolved hydrocarbons could affect the index of refraction for the resulting solution, solubilities of expected hydrocarbons are quite low (e.g., Cordier et al. 2013). Hence we do not expect for solutes to form more than $1 \%$ of the solution, and therefore their impact on the net index of refraction should be minimal.

Our calculation of $(I / F)_{\text {specular }}$ could also be affected by wave activity (Barnes et al. 2011), islands within the specular solar image footprint in the sea, or floating ice (Hofgartner \& 


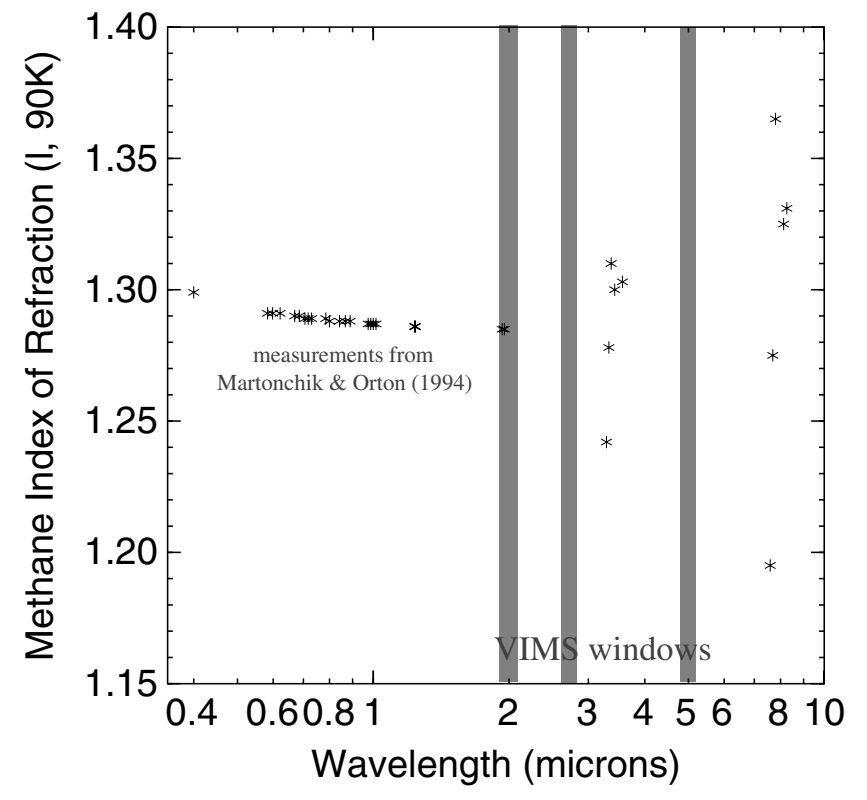

Figure 9. Measurements of the real index of refraction of methane liquid at $90 \mathrm{~K}$ from Martonchik \& Orton (1994). While we have not considered either ethane or trace solutes, the variability in the index of refraction with wavelength is small. Note that the imaginary index (absorption) does not enter into the calculation of specular reflectivity from Soderblom et al. (2012), and thus the reflectivity does not depend on absorption within the liquid.

Lunine 2013). An absolute photometric determination would also depend on an absolute calibration of the VIMS instrument to within $\sim 1 \%$ - much better than we can do with an instrument that has been in space for $15 \mathrm{yr}$ since its ground calibration. The original VIMS absolute radiometric calibration done on the ground was never more accurate than 10\%-15\% (see Brown et al. 2004).

Hence we choose $\tau_{\text {offset }}$ in order to be consistent with empirical estimates of the total optical depth in the $5 \mu \mathrm{m}$ channel. Vixie et al. (2013) fit multiple Titan observations of the same terrain types under different viewing conditions to estimate $\tau$ at $5 \mu \mathrm{m}$ to be 0.042 . Hence we choose $\tau_{\text {offset }}=0.691$ in order to force the $5 \mu \mathrm{m}$ optical depth to match that of Vixie et al. (2013). This ad hoc calibration is a source of systematic error-the entire $\tau$ curve could be shifted up or down if our choice of $\tau_{\text {offset }}$ is wrong.

We show our calculated optical depths in Figure 8, with the scale at left, inverted, with zero optical depth at the top. Since the number of points is relatively small, we also show the numerical values for optical depth in Table 1.

We calculate that the theoretical $(I / F)_{\text {specular }}$ for the parameters of the T85 flyby should be $\sim 70$ for a methane-rich lake and $\sim 90$ for an ethane-rich lake. Our empirical value for $(I / F)_{\text {specular }}$ is 32.4. While close, this value indicates some likely systematic errors in the absolute spectrophotometry, which warrants the artificial $\tau_{\text {offset }}$ correction.

\section{DISCUSSION}

Although direct comparison with VIMS Titan spectra requires a full radiative transfer treatment (beyond the scope of the present work), we can infer a few interesting points from the optical depths alone. The increasing optical depth with wavelength beyond $5 \mu \mathrm{m}$, for instance, implies that it may not be necessary to identify a solid surface absorber as responsible for the blue slope of Titan surface spectra longward of $5.0 \mu \mathrm{m}$.
Table 1

Measured One-way Optical Depths

\begin{tabular}{|c|c|}
\hline $\begin{array}{l}\text { Wavelength } \\
(\mu \mathrm{m}) \\
\lambda\end{array}$ & $\begin{array}{c}\text { Optical } \\
\text { Depth } \\
\tau\end{array}$ \\
\hline 1.969 & 1.685 \\
\hline 1.985 & 0.986 \\
\hline 2.001 & 0.798 \\
\hline 2.017 & 0.762 \\
\hline 2.034 & 0.775 \\
\hline 2.050 & 0.841 \\
\hline 2.067 & 1.001 \\
\hline 2.084 & 1.258 \\
\hline 2.646 & 1.598 \\
\hline 2.661 & 1.294 \\
\hline 2.681 & 0.995 \\
\hline 2.696 & 0.763 \\
\hline 2.712 & 0.909 \\
\hline 2.733 & 1.138 \\
\hline 2.748 & 1.038 \\
\hline 2.763 & 0.711 \\
\hline 2.781 & 0.503 \\
\hline 2.799 & 0.610 \\
\hline 2.816 & 0.985 \\
\hline 2.832 & 1.364 \\
\hline 2.849 & 1.271 \\
\hline 2.866 & 1.376 \\
\hline 2.882 & 1.406 \\
\hline 2.899 & 1.327 \\
\hline 2.915 & 1.292 \\
\hline 2.931 & 1.444 \\
\hline 4.869 & 0.314 \\
\hline 4.885 & 0.160 \\
\hline 4.902 & 0.113 \\
\hline 4.919 & 0.0676 \\
\hline 4.936 & 0.0624 \\
\hline 4.953 & 0.0434 \\
\hline 4.971 & 0.0420 \\
\hline 4.988 & 0.0432 \\
\hline 5.005 & 0.0572 \\
\hline 5.022 & 0.0549 \\
\hline 5.040 & 0.0664 \\
\hline 5.057 & 0.0738 \\
\hline 5.074 & 0.102 \\
\hline 5.091 & 0.151 \\
\hline 5.106 & 0.166 \\
\hline 5.123 & 0.189 \\
\hline
\end{tabular}

Note. These values are all modulo an assumed uniform offset factor $\tau_{\text {offset }}$ that fixes $\tau$ at $4.971 \mu \mathrm{m}$ to be 0.042 .

In general, it is not possible to disentangle the individual contributions to $\tau$ from $\tau_{\mathrm{ga}}, \tau_{\mathrm{hs}}$, and $\tau_{\mathrm{ha}}$ separately. The nearly straight line that can be drawn between the optical depth minima at the middle of the $2.0,2.8$, and $5 \mu \mathrm{m}$ windows probably reflects increasing haze cross section $\left(\tau_{\mathrm{hs}}+\tau_{\mathrm{ha}}\right)$ at shorter wavelengths. Increased haze scattering at shorter wavelengths is consistent with the Tomasko et al. (2008) model of $\sim 1 \mu \mathrm{m}$ sized haze particles.

The $2.7 / 2.8 \mu \mathrm{m}$ region is of particular interest for constraining surface composition, as the VIMS-measured $I / F$ values have been used in the past to rule out the presence of significant amounts of surface water ice (Clark et al. 2010). Our results show significant, if modest, increased $\tau$ at $2.7 \mu \mathrm{m}$ relative to 


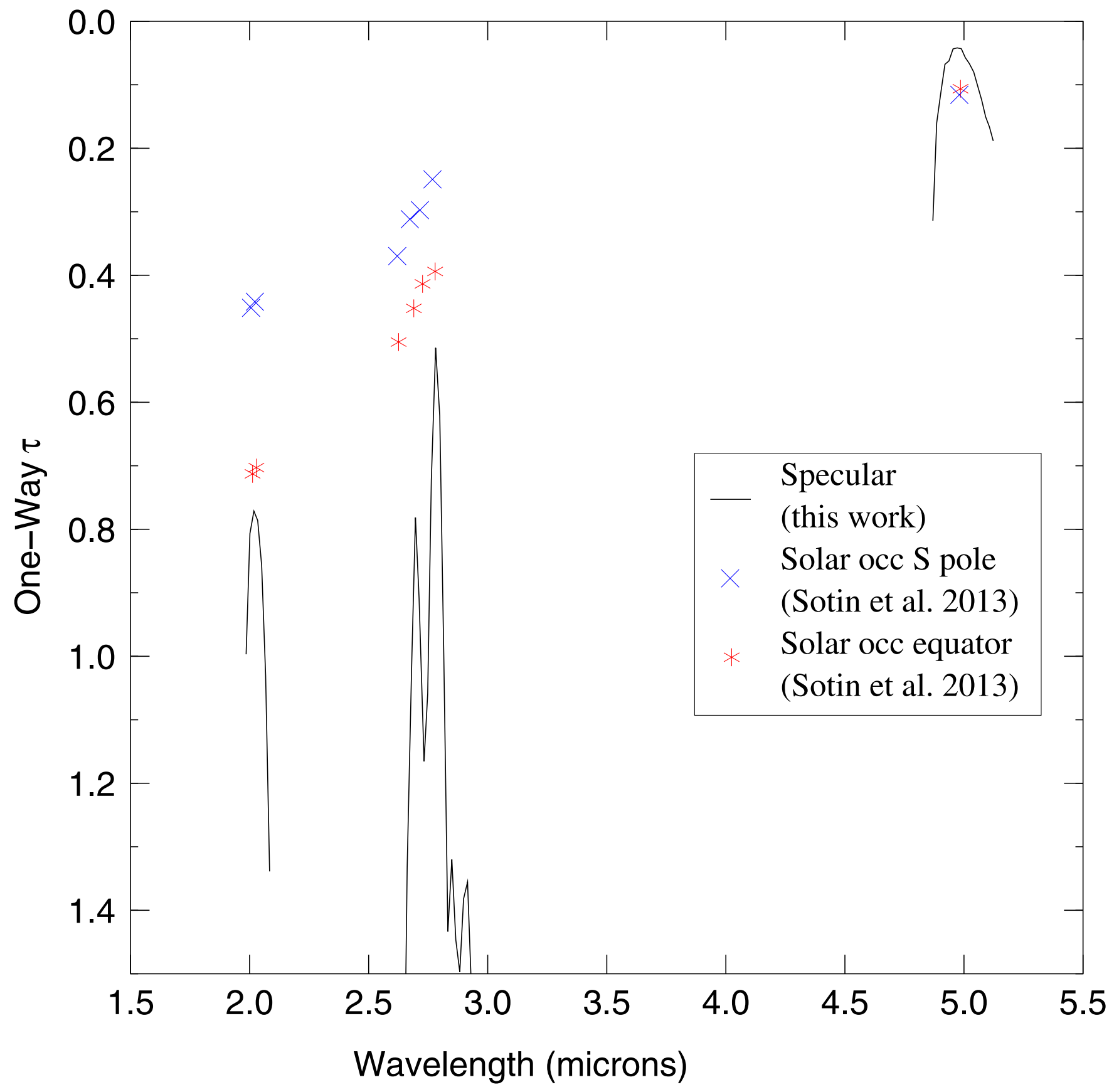

Figure 10. Comparison of our results for $\tau$ at the north pole with those at the south pole (blue $\times$ 's) and the equator (red *'s) derived from solar occultations by C. Sotin et al. (2013b, in preparation).

(A color version of this figure is available in the online journal.)

$2.8 \mu \mathrm{m}$-higher by a one-way $\Delta \tau=0.26$. The resulting ratio for $\tau_{2.7} / \tau_{2.8}$ is 1.52 , which is modestly higher than occultationderived values (1.2 as reported by Clark et al. 2010). Hence, a complete interpretation of the Titan $2.7 / 2.8 \mu \mathrm{m}$ flux ratio will require a radiative transfer model inversion to determine true surface albedos.

A much murkier window also seems to exist at $2.9 \mu \mathrm{m}$, though with a one-way optical depth near $\tau=1.0$. This window is likely affected by high atmospheric gas absorption $\tau_{\mathrm{ga}}$, rendering it incapable of providing significant surface information (though it is possible to see rough outlines of surface features in high-integration-time VIMS cubes with optimal viewing geometry). In general, light within the shorter wavelength windows preserves surface information, despite even higher total optical depths than that at $2.9 \mu \mathrm{m}$, due to the extremely high degree of forward-scattering from haze particles (Tomasko et al. 2008; Turtle et al. 2009).

The additional one-channel-wide peak that appears at $2.85 \mu \mathrm{m}$ in the transmission spectrum is suspicious because it does not appear brighter in haze scattering in typical Titan spectra. It may be a spurious cosmic-ray hit.

\subsection{Comparison with Solar Occultation}

Our transmission spectrum complements that determined using different methods. In particular, the technique known as solar occultation uses VIMS spectra of the Sun as Cassini passes 


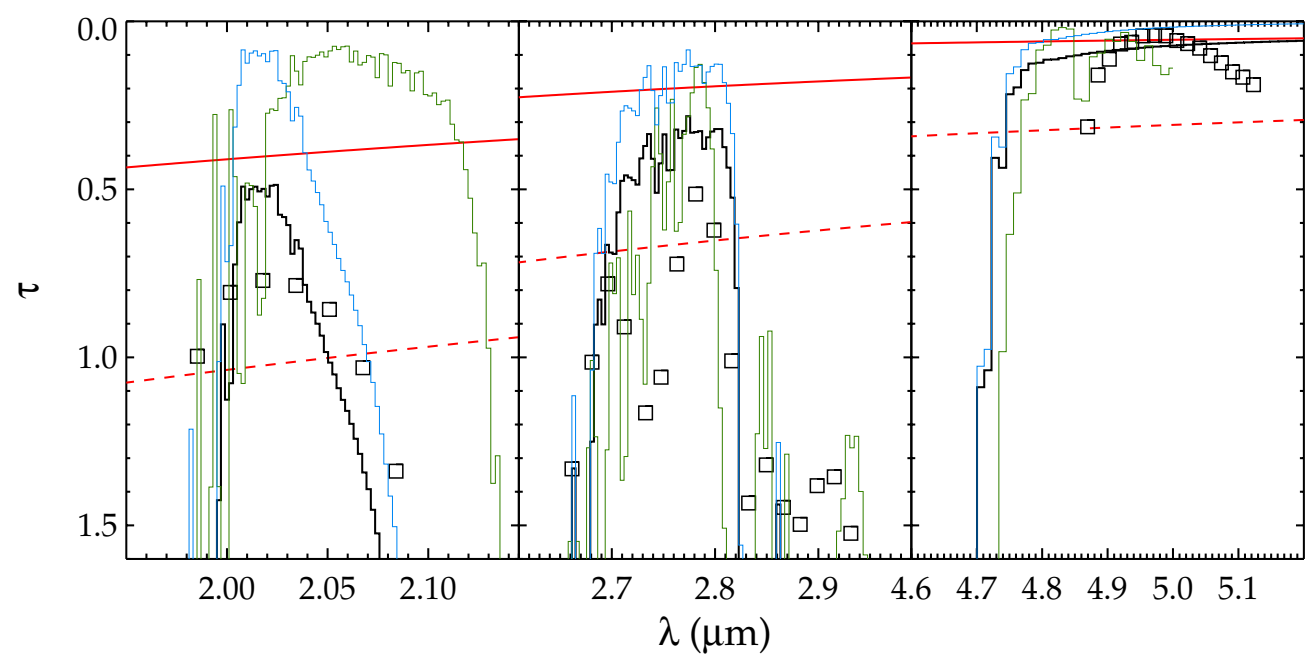

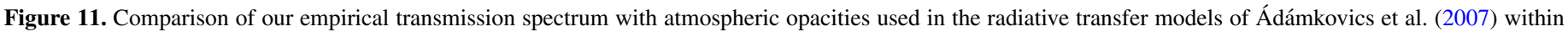

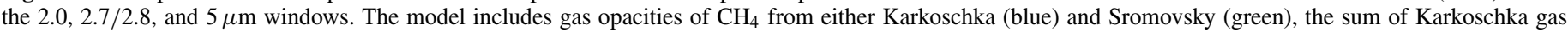

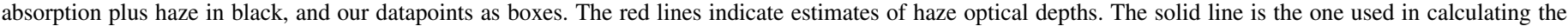
black haze-plus-gas curve, and the dashed line is a different plausible haze model with higher opacity for comparison.

(A color version of this figure is available in the online journal.)

behind Titan to infer a transmission spectrum. Solar occultations have the advantage of altitude resolution, meaning that they can be used to infer vertical distributions of haze, as was done by C. Sotin et al. (2013b, in preparation). Solar occultations can also be acquired at any latitude on Titan's surface, provided the appropriate spacecraft trajectory.

One relative advantage of our specular method is that it probes all the way to the surface while solar occultations generally cannot access the lower few kilometers of the atmosphere. This ability is of particular utility over Titan's lakes and seas, as it could be affected by vapors coming off the lake for any species, including methane, that is volatile enough to have a finite vapor pressure at the liquid's temperature. Another advantage is that this particular measurement was at the north pole $\left(87^{\circ} \mathrm{N}\right)$, while no solar occultations have previously been obtained northward of $45^{\circ} \mathrm{N}$. The north polar atmosphere is of particular interest given that nearly all Titan lakes are north of $60^{\circ}$ (Stofan et al. 2007), and given that most of the seasonal atmospheric changes take place in the arctic as well (e.g., Le Mouélic et al. 2012).

We show the equivalent one-way optical depths within Titan's atmospheric windows as gauged by solar occultations along with our results in Figure 10. Because of the free parameter $\tau_{\text {offest }}$ in our determination, the absolute mismatch between the measured $\tau$ values at $5 \mu \mathrm{m}$ is not meaningful. Had we adopted the C. Sotin et al. (2013b, in preparation) $5 \mu \mathrm{m} \tau$ as our calibration, then the entire black curve from Figure 10 would shift down by $\sim 0.07$.

The real discrepancy between the solar occultation and specular optical depths arises in the $2.7 / 2.8 \mu \mathrm{m}$ window. While the occultation measurements show an overall slope toward higher optical depth at $2.7 \mu \mathrm{m}$, similar to the specular result, the occultation does not show the strong absorption at $2.75 \mu \mathrm{m}$ that creates a valley between the 2.7 and $2.8 \mu \mathrm{m}$ subwindows. This difference may result from the solar occultation's inability to probe all the way down to the surface at these wavelengths. At $2 \mu \mathrm{m}$, which has a similar optical depth to $2.7 \mu \mathrm{m}$, the solar occultation can no longer see the Sun below $\sim 40 \mathrm{~km}$ altitude. Hence it is unable to see the troposphere, where $70 \%$ of the atmosphere and an even greater fraction of methane resides. Thus, the notch between 2.7 and $2.8 \mu \mathrm{m}$ might result from gaseous absorption in the lower atmosphere due to a volatile constituent (consistent with the partial identification of $\mathrm{CH}_{3} \mathrm{D}$ by Rannou et al. 2010, though the $\mathrm{CH}_{3} \mathrm{D}$ absorption is too narrow to produce the notch by itself).

\subsection{Comparison with Atmospheric Opacities}

Next, we compare our observed optical depths with predicted total optical depths from the atmospheric opacities used by Ádámkovics et al. (2007). Figure 11 shows the comparison.

From Figure 11 we see that our observed width of the $2 \mu \mathrm{m}$ window is greater than that predicted by the Ádámkovics model using the Sromovsky methane-line database, but smaller than those predicted when it uses the Karkoschka methane-line database. The VIMS spectral resolution is insufficient to see the fine-scale absorption structure predicted by the models.

The 2.7/2.8 window is again an enigma. The Ádámkovics model does not reproduce the double-peak structure with Sromovsky optical constants. The Karkoschka constants may do a better job, reproducing fairly well the $2.7 / 2.8$ ratio fairly well and reproducing the double-peak structure, too. In fact, given that we had to recreate the top of the 2.8 peak due to detector saturation, it is at least possible that we have overpredicted the $2.8 \mu \mathrm{m}$ absorption, which would place us even closer to the Karkoschka-constants model.

At $5 \mu \mathrm{m}$ both our and the Karkoschka constants agree on the blue end of the window, though ours do not increase again at $4.8 \mu \mathrm{m}$. The predictions use just methane absorption, while in reality carbon monoxide $(\mathrm{CO})$ absorption plays a strong role shortward of the blue edge of the $5 \mu \mathrm{m}$ window.

\subsection{Comparison with Radiative Transfer}

We used the Hirtzig et al. (2013) radiative transfer model to compute theoretical one-way normal optical depths for Titan's atmosphere in the $2,2.7 / 2.8$, and $5 \mu \mathrm{m}$ windows. The radiative transfer algorithm employs an atmospheric model grid with 70 layers extending from the surface up to $700 \mathrm{~km}$ altitude $\left(5 \times 10^{-5} \mathrm{mbar}\right.$ level $)$. For each atmospheric layer, the algorithm calculates the optical depths coming from gases and aerosols. 

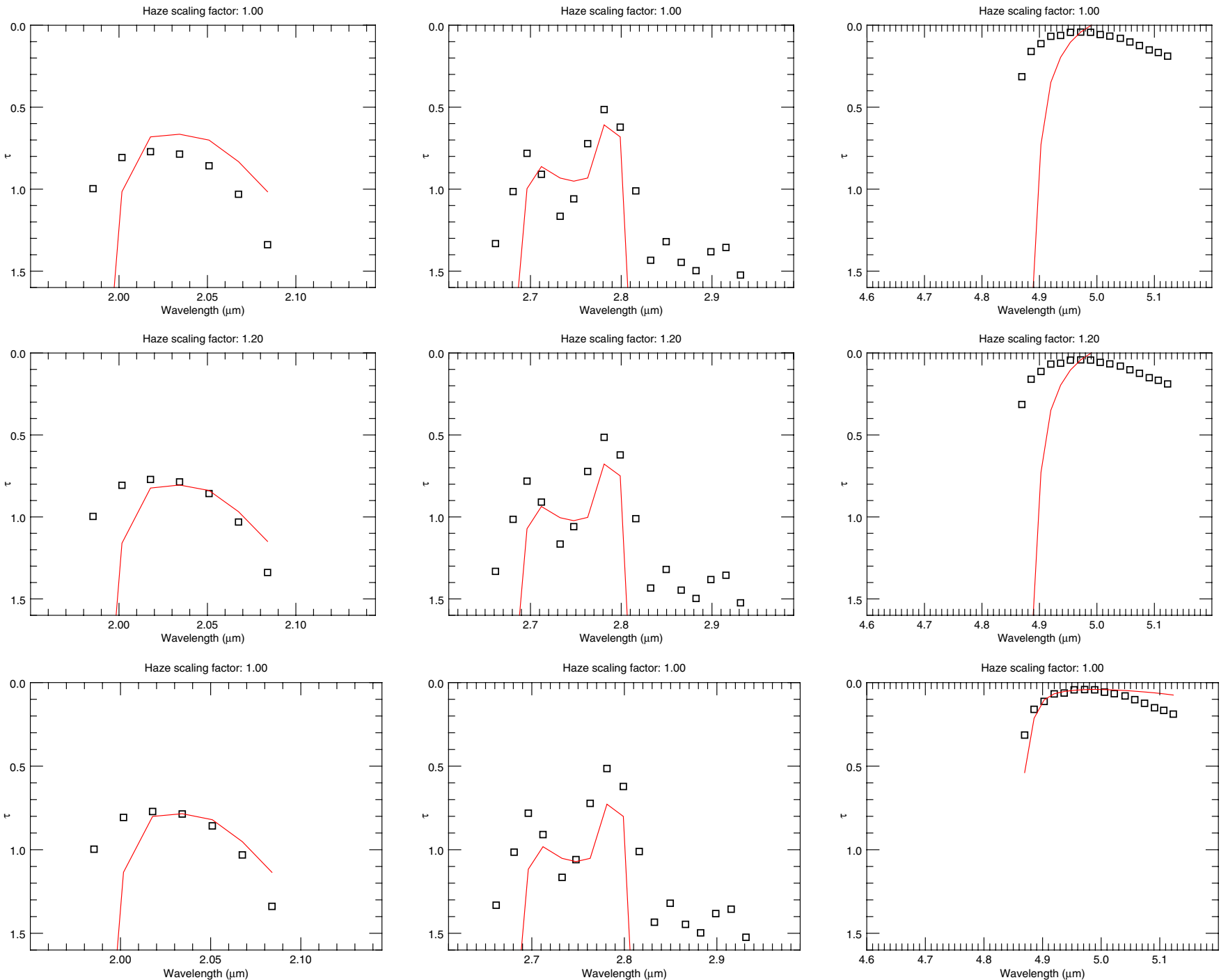

Figure 12. Comparison of the results of radiative transfer calculations (using the Hirtzig et al. 2013, model), shown in red, to our empirically derived specular optical depths, shown as the black squares. The left-hand column shows values within the $2 \mu \mathrm{m}$ window, the middle column the $2.7 / 2.8 \mu \mathrm{m}$ double window, and the right-hand column the $5 \mu \mathrm{m}$ window. The top row shows radiative transfer calculations that assume the same haze structure as observed by Huygens. The middle row achieves a better fit using an enhanced amount of haze- $120 \%$ of the Huygens value. The bottom row is an aggressively tuned model that manages a better fit to the observations with normal haze but just $10 \%$ of the nominal carbon monoxide concentration.

(A color version of this figure is available in the online journal.)

The gaseous contributors to Titan's atmospheric opacity include $\mathrm{N}_{2}-\mathrm{N}_{2}$ and $\mathrm{N}_{2}-\mathrm{H}_{2}$ collision-induced absorptions (McKay et al. 1989; Lafferty et al. 1996), Rayleigh scattering from $\mathrm{N}_{2}$ and $\mathrm{CH}_{4}$ (Peck \& Khanna 1966; Weber 2002), and absorption from gaseous ${ }^{12} \mathrm{CH}_{4},{ }^{13} \mathrm{CH}_{4}, \mathrm{CH}_{3} \mathrm{D}$ and $\mathrm{CO}$. Between 1.71 and $5.12 \mu \mathrm{m}$, up-to-date line parameters of methane and its isotopologues are taken from Boudon et al. (2006), Albert et al. (2009), and Nikitin et al. (2002, 2006, 2013). CO line parameters come from the GEISA 2009 database (Jacquinet-Husson et al. 2011). Those molecular line parameters were used to calculate $k$-correlated coefficients for each VIMS infrared channel within this spectral range, considering (1) the pressure-temperature grid defined from the Huygens/HASI measurements (Fulchignoni et al. 2005), (2) the methane vertical profile derived by Niemann et al. (2010) from Huygens Gas Chromatograph Mass Spectrometer measurements, (3) a uniform CO mole fraction equal to $3.2 \times 10^{-5}$ based on Cassini/VIMS measurements (Baines et al. 2006), and (4) $\mathrm{D} / \mathrm{H}$ and ${ }^{12} \mathrm{C} /{ }^{13} \mathrm{C}$ isotopic ratios of $1.3 \times 10^{-4}$
(Bézard et al. 2007) and $88.5 \pm 1.4$ (Mandt et al. 2012) respectively.

Aerosol optical depth profiles derive from the aerosol extinction profile calculated by Tomasko et al. (2008). This profile was measured during the descent of the Huygens probe at a single time (southern summer) and location (near the equator). Therefore, it may not be fully representative of the aerosol extinction profile of the north polar region of Titan at the time of the T85 flyby (northern spring). A uniform scaling factor for the Tomasko et al. (2008) aerosol extinction profile is thus used to take into account latitudinal and seasonal variations of Titan's haze opacity.

The one-way normal optical depth of Titan's atmosphere is obtained by summing the opacity of gases and aerosols over each atmospheric layer.

The one-way normal optical depth $\left(\tau_{\text {model }}\right)$ computed with the radiative transfer model of Hirtzig et al. (2013) is compared with the one inferred from the VIMS observation of the specular reflection over Kivu Lacus ( $\tau_{\text {obs }}$ ) (Figure 12), using the same 
normalization (i.e., using a constant $\tau_{\text {offset }}$ to fix the optical depth at $4.971 \mu \mathrm{m}$ at 0.042 ).

Considering a nominal case for the haze extinction profile (scaling factor for the Tomasko et al. 2008 aerosol extinction profile equal to 1), the Hirtzig model globally predicts atmospheric optical depths in better agreement (in shape and magnitude) with the optical depths inferred from the observation than do the Ádámkovics opacities from Figure 11). The width of the $2 \mu \mathrm{m}$ window and the double-peak structure of the $2.7 / 2.8 \mu \mathrm{m}$ window are both better reproduced, and the optical depth of the blue edge of the $5 \mu \mathrm{m}$ window is larger (thanks to the incorporation of the $\mathrm{CO}$ absorption-not included in the Adamkovics model). Yet, the optical depth given by the Hirtzig model is too low at $2 \mu \mathrm{m}$, and too high in the 2.7 and $2.8 \mu \mathrm{m}$ window peaks.

By varying the amount of haze in the model, it is possible to get a better fit. The best overall fit is obtained by scaling the Tomasko et al. (2008) aerosol extinction profile by a factor of 1.2, keeping all other atmospheric parameters constant (Figure 12, middle row). The red edge of the $2 \mu \mathrm{m}$ window matches perfectly $\tau_{\text {obs }}$ in this spectral region. The modeled optical depth reproduces well the shape of $\tau_{\text {obs }}$ in the $2.7 / 2.8 \mu \mathrm{m}$ windows, but the modeled optical depth of the two peaks and of the spectral region in between the peaks is still too large. The increased haze over the northern polar region ( $20 \%$ greater than that of the equatorial region at northern winter season) may be explained by leftover haze from a lingering winter polar hood.

The modeling is still not perfect. In all cases, the plateau of relatively low optical depths at wavelengths longer than $2.8 \mu \mathrm{m}$ is not adequately reproduced. Neither is the blue wing of the $5 \mu \mathrm{m}$ window (common "known" flaws of Titan radiative transfer models, due to the lack of good enough line parameters in those spectral regions and, maybe, in our case, of systematic errors in reconstructing the 2.8 and $5 \mu \mathrm{m}$ saturated signals). The precise shape of the far wing of the $2.7 / 2.8 \mu \mathrm{m}$ double window is still far from being fully understood (even for unsaturated spectra).

Setting aside the $2.7 / 2.8 \mu \mathrm{m}$ double window, we can fit the observations better with a more aggressively tuned model (and perhaps overtuned). As we show in Figure 12 in the bottom row, we manage to better fit the $5 \mu \mathrm{m}$ window by drastically reducing the amount of $\mathrm{CO}$ (to $1 / 10$ of its nominal value, down to $3.2 \mathrm{ppm}$ ) and fixing the haze scaling factor at a value of 1.0. The fit to the $5 \mu \mathrm{m}$ windows is now much improved, but the $\mathrm{CO}$ content is at odds with prior expectation, and hence perhaps unlikely.

\section{CONCLUSION}

A specular reflection observed on T85 is bright enough to be seen at 2.8, 2.7, and $2.0 \mu \mathrm{m}$ as well as $5 \mu \mathrm{m}$. We have used the relative intensity of the specular reflection to infer an empirical transmission spectrum of Titan's atmosphere in multiple channels within those atmospheric windows.

Ours is the first measurement of Titan's atmospheric conditions near the north pole. The specular-measured optical depth at $2 \mu \mathrm{m}$ is between that of the south pole and the equator as determined by solar occultations. We show an increased optical depth at $2.7 \mu \mathrm{m}$ relative to that at $2.8 \mu \mathrm{m}$, with a strong atmospheric absorption in between. The behavior of atmospheric transmission in the $2.7 / 2.8 \mu \mathrm{m}$ region suggests caution when using this ratio to interpret surface composition.

The prospects for deconvolving the surface and atmospheric contributions to Titan's overall near-infrared spectrum motivate this work. When comparing our optical depths to those predicted by radiative transfer algorithms, our work confirms known areas of deficiency such as the wings of the $2 \mu \mathrm{m}$ window and the shape of the $2.7 / 2.8 \mu \mathrm{m}$ window. Our empirical result will help drive improvements in future models.

The spectacular nature of the T85 Kivu specular reflection results from Cassini's proximity to Titan at the time of the observation $(33,000 \mathrm{~km})$. Additional observations of this class would allow monitoring of Titan's north polar atmosphere as it transitions toward northern summer. Future observations from even lower altitudes might allow extension of the derived transmission spectrum down to the $1.6 \mu \mathrm{m}$ window. Because Huygens DISR data exist at $1.6 \mu \mathrm{m}$, such an observation would allow direct comparison with results obtained from the Huygens landing site, and would inform the extrapolation of the DISR results to longer wavelengths.

Future planners should consider the high intensity of specular reflections when designing future observations. In order to avoid saturation, alternating cubes of long $(80 \mathrm{~ms})$ and short $(13 \mathrm{~ms})$ duration might lead to fewer systematic errors due to the reconstruction of saturated pixels.

In the very long term, observations of specular reflections off of extrasolar planet oceans, as considered by Robinson et al. (2010), could also be used to quantify the transmission spectrum of their atmospheres. Such a measurement would require direct detection of the planet. But specular atmospheric transmission could complement direct whole-planet spectroscopy (e.g., Tinetti et al. 2006) and transmission spectroscopy in transit (e.g., Seager \& Sasselov 2000; Brown 2001; Hubbard et al. 2001; Charbonneau et al. 2002) in a similar fashion to its use at Titan.

The authors acknowledge the support of the NASA/ESA Cassini mission. J.W.B. acknowledges support from the NASA CDAPS Program, grant No. NNX12AC28G. M.Á. is supported in part by NSF planetary astronomy grant AST-1008788. T.A. and S.R. benefited from the support by the Agence Nationale de la Recherche (ANR projects "APOSTIC" No. 11BS56002, France).

\section{REFERENCES}

Ádámkovics, M., de Pater, I., Hartung, M., \& Barnes, J. W. 2009, P\&SS, 57,1586

Ádámkovics, M., Wong, M. H., Laver, C., \& de Pater, I. 2007, Sci, 318, 962

Albert, S., Bauerecker, S., Boudon, V., et al. 2009, CP, 356, 131

Badoz, J., Le Liboux, M., Nahoum, R., et al. 1992, RScI, 63, 2967

Bailey, J., Ahlsved, L., \& Meadows, V. S. 2011, Icar, 213, 218

Baines, K. H., Drossart, P., Lopez-Valverde, M. A., et al. 2006, P\&SS, 54, 1552

Barnes, J. W., Brown, R. H., Soderblom, L., et al. 2007, Icar, 186, 242

Barnes, J. W., Soderblom, J. M., Brown, R. H., et al. 2011, Icar, 211, 722

Bellucci, A., Sicardy, B., Drossart, P., et al. 2009, Icar, 201, 198

Bézard, B., Nixon, C. A., Kleiner, I., \& Jennings, D. E. 2007, Icar, 191, 397

Boudon, V., Rey, M., \& Loëte, M. 2006, JQSRT, 98, 394

Brown, R. H., Baines, K. H., Bellucci, G., et al. 2004, SSRv, 115, 111

Brown, R. H., Soderblom, L. A., Soderblom, J. M., et al. 2008, Natur, 454, 607

Brown, T. M. 2001, ApJ, 553, 1006

Charbonneau, D., Brown, T. M., Noyes, R. W., \& Gilliland, R. L. 2002, ApJ, 568,377

Clark, R. N., Curchin, J. M., Barnes, J. W., et al. 2010, JGRE, 115, 10005

Cordier, D., Barnes, J., \& Ferreira, A. 2013, Icar, 226, 1431

Fulchignoni, M., Ferri, F., Angrilli, F., et al. 2005, Natur, 438, 785

Griffith, C. A., Doose, L., Tomasko, M. G., Penteado, P. F., \& See, C. 2012, Icar, 218,975

Hirtzig, M., Bézard, B., Lellouch, E., et al. 2013, Icar, 226, 470

Hofgartner, J. D., \& Lunine, J. I. 2013, Icar, 223, 628

Hubbard, W. B., Fortney, J. J., Lunine, J. I., et al. 2001, ApJ, 560, 413

Jacquinet-Husson, N., Crepeau, L., Armante, R., et al. 2011, JQSRT, 112, 2395 
Lafferty, W. J., Solodov, A. M., Weber, A., Olson, W. B., \& Hartmann, J.-M. 1996, ApOpt, 35, 5911

Le Mouélic, S., Rannou, P., Rodriguez, S., et al. 2012, P\&SS, 60, 86

Lellouch, E., Coustenis, A., Sebag, B., et al. 2003, Icar, 162, 125

Mandt, K. E., Waite, J. H., Teolis, B., et al. 2012, ApJ, 749, 160

Martonchik, J. V., \& Orton, G. S. 1994, ApOpt, 33, 8306

McKay, C. P., Pollack, J. B., \& Courtin, R. 1989, Icar, 80, 23

Niemann, H., Atreya, S., Demick, J., et al. 2010, JGR, 115, E12006

Nikitin, A., Brown, L., Fejard, L., Champion, J., \& Tyuterev, V. G. 2002, JMoSp, 216,225

Nikitin, A., Brown, L. R., Sung, K., et al. 2013, JQSRT, 114, 1

Nikitin, A., Champion, J.-P., \& Brown, L. R. 2006, JMoSp, 240, 14

Peck, E. R., \& Khanna, B. N. 1966, JOSA, 56, 1059

Rannou, P., Cours, T., Le Mouélic, S., et al. 2010, Icar, 208, 850

Robinson, T. D., Meadows, V. S., \& Crisp, D. 2010, ApJL, 721, L67
Rodriguez, S., Le Mouélic, S., Sotin, C., et al. 2006, P\&SS, 54, 1510

Seager, S., \& Sasselov, D. D. 2000, ApJ, 537, 916

Soderblom, J. M., Barnes, J. W., Soderblom, L. A., et al. 2012, Icar, 220, 744

Stephan, K., Jaumann, R., Brown, R. H., et al. 2010, GeoRL, 37, L7104

Stofan, E. R., Elachi, C., Lunine, J. I., et al. 2007, Natur, 445, 61

Teanby, N. A., Irwin, P. G. J., de Kok, R., \& Nixon, C. A. 2010, ApJL, 724, L84

Tinetti, G., Meadows, V. S., Crisp, D., et al. 2006, AsBio, 6, 34

Tomasko, M. G., Archinal, B., Becker, T., et al. 2005, Natur, 438, 765

Tomasko, M. G., Doose, L., Engel, S., et al. 2008, P\&SS, 56, 669

Turtle, E. P., Perry, J. E., McEwen, A. S., et al. 2009, GeoRL, 36, L2204

Vinatier, S., Bézard, B., Fouchet, T., et al. 2007, Icar, 188, 120

Vixie, G., Barnes, J. W., Bow, J., et al. 2012, P\&SS, 60, 52

Vixie, G., Barnes, J. W., Jackson, B., \& Wilson, P. 2013, JGRE, submitted

Weber, M. J. 2002, Handbook of Optical Materials (Boca Raton, FL: CRC Press) 